\title{
Research of SNMP-based network topology discovery algorithm in IOT Minglang Yang ${ }^{1,}$, Chuanchang Liu $^{2, b}$ \\ ${ }^{1}$ State Key Laboratory of Networking and Switching Technology, Beijing University of Posts and Telecommunications, Beijing 100876, China. \\ ${ }^{2}$ State Key Laboratory of Networking and Switching Technology, Beijing University of Posts and Telecommunications, Beijing 100876, China.
}

ayangminglang816@163.com, ${ }^{\mathrm{b}}$ Icc3265@126.com

Keywords: IOT, device auto-discovery, network management, topology discovery.

\begin{abstract}
Due to the rapid development of the IOT, more and more devices in the network need to be connected to the IOT. Device management and device auto-discovery has become increasingly important. Network topology is a way to describe the logical or physical connections between network devices, and it is extremely necessary for the configuration management, even for the entire network management. Because of the dynamic and the increasing scale of the networks, getting topology manually is almost impossible. As a result, the researchers begin to study the network topology auto-discovery technology. For the reasons of more and more network equipment providing $S N M P$ protocol, a $S N M P$-based topology discovery algorithm is brought forward. This paper first research the $S N M P$-based topology discovery algorithm. Then on the base of the indicators refined for topology discovery algorithms, we compare several topology discover methods and sum up the most suitable application environment for the SNMP-based algorithm. Finally this paper provides the details of implementation of the SNMP-based topology discovery tool, including the implementation of the algorithm and the architecture and the mutual flow of the software.
\end{abstract}

\section{Introduction}

In recent years, due to the rapid development of computer networks, effective network management has been more and more attention, and accessing the latest network topology structure is important to network management [1]. A good network management system must be aware of the topology of the entire network. Besides, due to the rapid development of the IOT, more and more devices in the network need to be connected to the IOT. Device management and device auto-discovery has become increasingly important. Network topology is an important basic information of computer network, which is the basis of network management, data simulation and information gathering. Topology discovery can effectively help network management, also topology discovery results can be widely applied to the device management and device auto-discovery. Based on the above reasons, the research of network topology discovery technology is very important. The topology discovery in network management is to obtain and maintain the information of the existence of network elements and the connection between them, then achieve effective management of network.

\section{Key technologies}

\subsection{SNMP.}

Simple network management protocol (SNMP) is the most widely used network management protocol in TCP/IP network. In May 1990, RFC1157 defined the first version of SNMP (simple network management protocol) called SNMPv1. RFC1157 and another file RFC1155, provide a method for monitoring and managing computer networks. Because SNMP has the following characteristics, SNMP has been widely used, and has become the standard of network management: Easy to implement. Open standards, complete technical documentation. Based on the UDP, do not rely too much on the agreement, and will not increase the burden of management. 


\subsection{MIB.}

The basic idea of SNMP is that all network devices maintain a management information base (MIB) to keep the relevant information of all the running processes and to respond to the query of the management workstation [2]. Each managed device which support SNMP keeps a number of MIB libraries.

The management information base $(M I B)$ specify the variables maintained by the network elements, and provide the data structure of the collection of all the possible managed objects in the network. All the statistical contents of the gateway can be obtained by accessing these data items. $M I B-I I$ is the second edition of $M I B$, which is the extension of it. Network topology discovery is to find the connection between the router and the subnet, the information source is the MIB-II library defined by SNMP. The structure of MIB is shown in Fig.1.

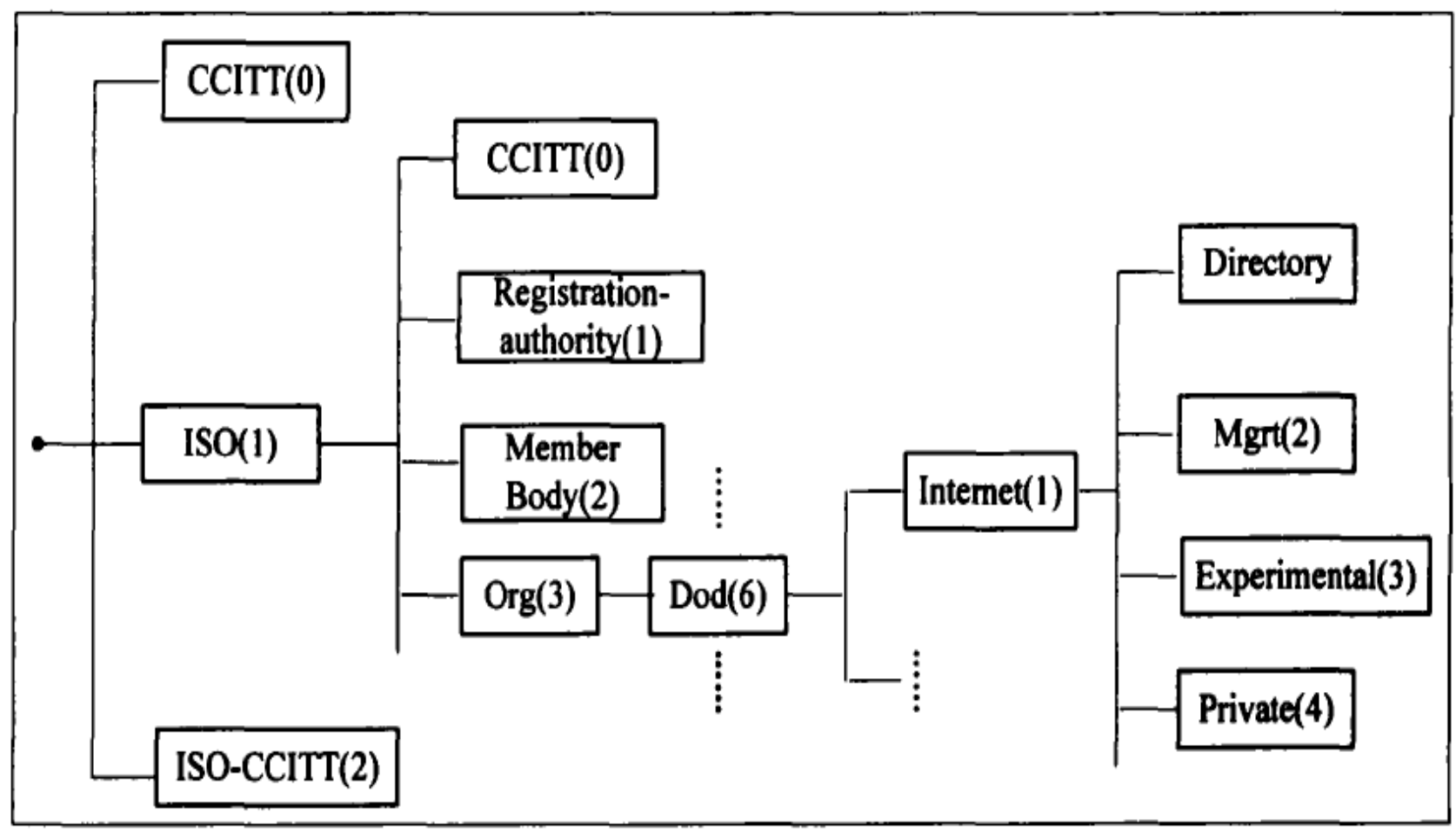

Fig. 1 The structure of $M I B$

The MIB-II information about router level topology discovery is divided into 3 groups: system group, interface group and IP group.

System group. Including 7 simple variables, sysservice can be used to determine the type of device, from the lowest to seventh in its binary form, if a bit is 1 then provide service of OSI corresponding level. The third bit is 1 shows that the node provides routing function and is a router device.

Interface group. Contains general information about the physical interface of the entity, including the configuration information and the statistical information that occurs at each interface [3]. It defines a simple variable ifnumber, which represents the number of the device interfaces, and an interface table iftable, one line for each interface. Index represents the index terms of the table, whose values range from 1 to ifnumber, and assign a unique serial number for each interface. fdescr is the interface name, iftype is the interface type, which is the type of the sub network of the interface, such as Ethernet, 802.5 token ring, FDDI. It can be seen that the iftable of router interface table shows the simple topology of the connected sub network.

$I P$ group. The IP group defines a number of simple variables. ifforwarding is 1 indicates that the node has a IP forwarding function, which represents this node is a router. This group also defines 3 very useful table variables: IP address table, IP routing table, and ARP address translation table [4]. They are important information sources for network topology discovery. 


\section{Algorithm Research}

The key to Topology discovery technology is capturing the network topology information. The difficulty is to find the place where the effective topology information store, and to find the way to get these information legally. SNMP protocol is a good solution to these two difficulties.

The topology of the network is the connection relation of the sub network. The division of the sub networks is based on the IP address of the terminal equipment. The common features between the IP addresses in the sub networks are called sub networks [5]. The connection between the sub networks is achieved by the routing device, and a sub network is connected to a port on a router device. Also, routing devices can be interconnected. The scheme of network topology is shown in Fig. 2.

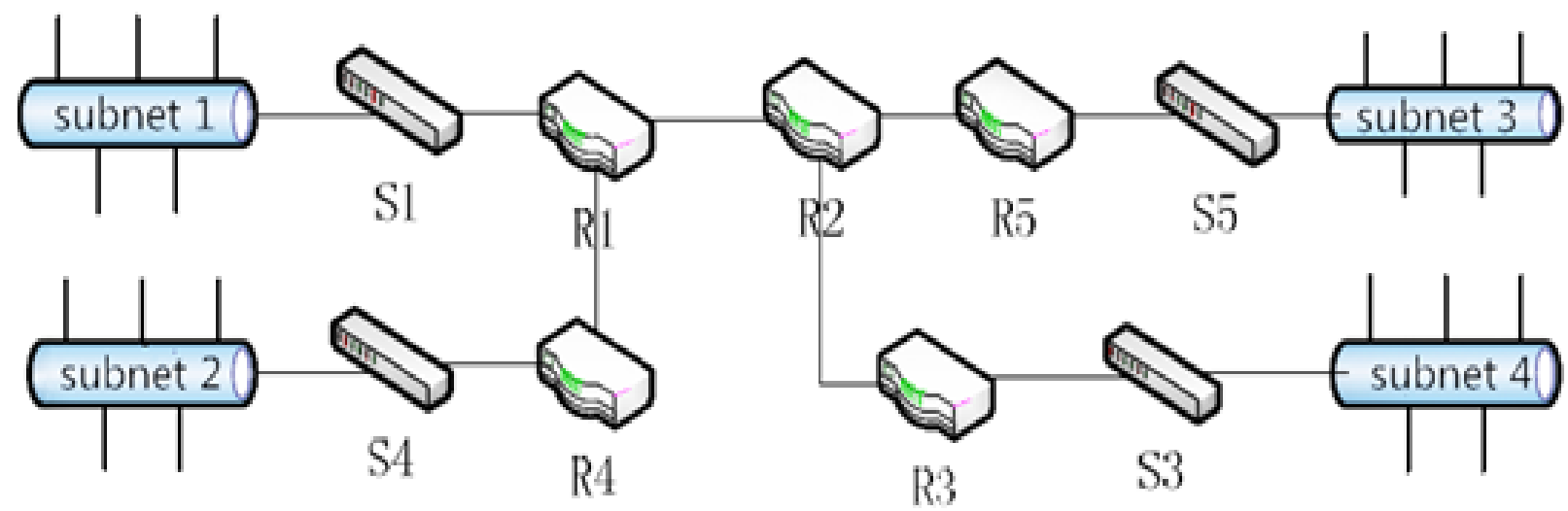

Fig.2 The scheme of network topology

According to the characteristics of the SNMP protocol, we access the list of routing devices or the list of hosts from MIB-II information in routers or VLAN-MIB, Bridge-MIB information in switches, bridges through the SNMP protocol, these information are basic information to construct network topology. Based on these information, we can use breadth first search algorithm to construct the three list: the Unchecked routing equipment, topology information chain, subnet information list. Unchecked routing equipment list stores the known but not scanned routing devices; Topology information list stores the scanned routing devices and the sub network connected to these devices. The scan process is to obtain the sub network of the device and the unchecked routing equipment; Sub network information linked list stores the information of the scanned sub network, including the device condition under the sub network.

Topology discovery algorithm based on SNMP can be described as such a process: use a seed router, access all the reachable segment from its routing table records, and the next hop router port IP address and the related routing information, then it will continue to expand its search, until reaching the user specified depth. Also it can obtain directly connected subnet and its subnet mask of all ports on each routing equipment, according to the information, and then get all the active hosts in the subnets. If these devices support SNMP, the system and IP address information can be collected.

In short, as long as the IP address of the seed router is given (usually using the IP address of the local gateway as the address of the seed router), the network topology information of all routing devices and active hosts in the specified depth can be obtained.

\section{Summary}

At present, most routers have shielded the broadcast ping, the ICMP netmask request packet, and DNS Zone Transfer detecting packet. And SNMP is the most commonly used environmental management agreement.

SNMP's biggest advantage is that the network status information is automatically updated, and almost all network equipment support SNMP. The topology information obtained by SNMP is always able to objectively and accurately reflect the latest status of the network. In addition, the SNMP is designed to be independent of the protocol, so it can be used in IP, IPX, AppleTalk, OSI, etc., which greatly improves the compatibility of the network environment and the general applicability of the 
topology discovery tool. These advantages ensure the accuracy and real-time of the topology information, and will not bring too much network load, which is in line with the actual needs of small and medium network topology discovery.

Because of the complexity and heterogeneity of the network structure itself, any single network topology discovery method can not have a good generality. In order to improve the accuracy of the results, and to provide a reliable analytical basis for all kinds of research based on network topology discovery, we should combine several methods, and analyze and mine the information that we collected. However, for the general research and application, especially in small and medium size networks, the network topology based on SNMP protocol has the incomparable advantages.

\section{Acknowledgments}

The work was supported by the National Natural Science Foundation of China (Grant No.U1536112).

\section{Reference}

[1]. Nini Yang, Xinhui Zhao, et al. SNMP based Web network management. Micro computer information, 2006 (2-3): 110-112.

[2]. Yupeng Li, Huanzhao Wang, et al. Network topology discovery based on SNMP and Java. Computer engineering and applications, 2004, 40 (5): 152-154.

[3]. Xiankun Ren, Jiahai Yang, Peiyu Wang, et al. Network management theory and Implementation Technology (First Edition). Tsinghua University press, 2000, p. 122-168.

[4]. Yingcai Bai, et al. The design and application of computer network management system. Tsinghua University press, 1999, p. 245-289.

[5]. Xiandao Cen, Changqing An, et al. Network management protocol and application development. Tsinghua University press, 1998, p. 86-164. 